\title{
GMR
}

\section{High levels of glucose promote the activation of hepatic stellate cells via the p38-mitogen- activated protein kinase signal pathway}

\author{
L.K. Wu, Y.C. Liu, G. Ma, L.L. Shi and X.M. He \\ The Second Clinical College, Zhejiang TCM University, Gongshu District, \\ Hangzhou, China \\ Corresponding author: X.M. He \\ E-mail: wulingkang@163.com \\ Genet. Mol. Res. 15 (3): gmr.15038419 \\ Received January 11, 2016 \\ Accepted May 17, 2016 \\ Published August 29, 2016 \\ DOI http://dx.doi.org/10.4238/gmr.15038419 \\ Copyright $(2016$ The Authors. This is an open-access article distributed under the terms of \\ the Creative Commons Attribution ShareAlike (CC BY-SA) 4.0 License.
}

\begin{abstract}
The relationship between the p38-mitogen-activated protein kinase (p38-MAPK) signal pathway and high glucose-induced hepatic stellate cell (HSC) activation was investigated in this study. Sixty human HSC samples were randomly selected and used in the control (cultured normally), high-glucose (cultured in the presence of high glucose), and blocking (cultured under high-glucose conditions in the presence of the p38-MAPK inhibitor, SB203580) groups. The cells were incubated for $120 \mathrm{~h}$ and subsequently analyzed for morphological changes by inverted microscopy and for $\alpha$-smooth muscle actin ( $\alpha$-SMA) expression (to determine the degree of HSC activation) by the method of streptavidin-biotin complex and western blot. Phospho-p38MAPK protein expression was analyzed by western blotting. $\alpha$-SMA and phospho-p38-MAPK expression was significantly upregulated in HSCs cultured under high-glucose conditions, compared to the HSCs cultured normally $(\mathrm{P}<0.01)$. On the other hand, phospho-p38-MAPK
\end{abstract}


and $\alpha$-SMA protein levels were significantly lower in the blocking group compared to the high-glucose group $(\mathrm{P}<0.01)$. Based on these results, we concluded that high-glucose levels induce HSC activation mediated by phospho-p38-MAPK. Therefore, blocking the p38-MAPK signal pathway could inhibit this effect.

Key words: Hepatic stellate cells; $\alpha$-Smooth muscle actin; Glucose; p38-Mitogen-activated protein kinase signaling pathway; SB203580

\section{INTRODUCTION}

Hepatic stellate cells (HSCs) are the main effector cells of liver fibrosis (Friedman, 2008a; Li et al., 2008). HSC activation and the resulting excessive deposition of extracellular matrix is the central feature of liver fibrosis (Friedman, 2008b); therefore, prevention of HSC activation can affect the development of liver fibrosis (De Minicis et al., 2007; Friedman, 2008c). However, the regulation of HSC activation is a highly complex process. Recent studies have shown that the p38-mitogen-activated protein kinase (p38-MAPK) signaling pathway plays a very important role in HSC stress, activation, proliferation, apoptosis, phenotypic transdifferentiation, and other physiological and pathological processes (Brown and Sacks, 2008; Kyriakis and Avruch, 2012). In this study, the relationships between the p38-MAPK signaling pathway, HSC activation, and liver fibrosis were further elucidated by identifying changes in p38-MAPK signaling in HSCs activated by high-glucose levels in culture. A reduction in HSC activation corresponding to the blockage of the p38-MAPK signaling pathway further supported the existence of a close relationship between p38-MAPK signaling and HSC activation.

\section{MATERIAL AND METHODS}

\section{Cell lines and reagents}

The LI-90 HSCs were obtained from the Experimental Animal Center of Sun Yatsen University (Guangzhou, China). Dulbecco's modified Eagle's medium with L-glutamine (L-DMEM) was purchased from Gibco (Thermo Fisher Scientific, Waltham, MA, USA). Fetal calf serum was purchased from Harry Bio-Engineering Co., Ltd. (Chengdu, China). P38-MAPK inhibitor SB203580 was purchased from New England Biolabs (Ipswich, MA, USA). Primary antibodies against $\alpha$-smooth muscle actin ( $\alpha$-SMA), phospho-p38-MAPK, and GAPDH, and their corresponding secondary antibodies were purchased from Boster BioEngineering Co., Ltd. (Wuhan, China). The ECL kit was purchased from KGI Bio-Technology Development Co., Ltd. (Nanjing, China) and liraglutide was purchased from Novo Nordisk (Bagsvaerd, Denmark).

\section{Grouping}

Sixty randomly selected human HSC samples were equally divided into 3 experimental groups: control, high-glucose, and blocking ( $\mathrm{N}=20$ each). Samples belonging to the control group were cultured in a routine manner. Samples in the high-glucose group were cultured in

Genetics and Molecular Research 15 (3): gmr.15038419 
a medium supplemented with glucose to give a final concentration of $25 \mathrm{mM}$, and samples in the blocking group were cultured in high-glucose medium (concentration $=25 \mathrm{mM}$ ) in the presence of the p38-MAPK inhibitor SB203580 (concentration $=40 \mu \mathrm{M}$ ). All samples were incubated for $120 \mathrm{~h}$.

\section{Experimental methods}

\section{HSC culture}

HSCs were recovered, seeded in $2.5-\mathrm{mL}$ plastic culture flasks, and cultured in L-DMEM supplemented with $10 \%$ (volume fraction) fetal bovine serum in a $5 \%$ (volume fraction) $\mathrm{CO}_{2}$ humidified incubator (He Cheng Instrument, Shanghai, China) at $37^{\circ} \mathrm{C}$. The medium was changed every 3 days.

\section{Analysis of HSC morphology}

The HSC morphology was analyzed under an inverted microscope (Olympus Corporation, Tokyo, Japan). Activated HSCs appeared flat with large cell bodies and welldeveloped stress fibers. Moreover, these cells appeared to lack cytoplasmic lipid droplets.

\section{Detection of HSC activation by immunocytochemistry}

HSC activation is characterized by the expression of $\alpha$-SMA. The extent of HSC activation can be determined by detecting $\alpha$-SMA expression in situ using the streptavidin-biotin complex (SABC) method. The samples were fixed with $2.5 \mathrm{~mL} 4 \%$ paraformaldehyde for $15 \mathrm{~min}$. The medium was aspirated and the samples were rinsed twice with phosphate-buffered saline (PBS) for 5 min each. Each sample was treated with a 1:50 mixture of hydrogen peroxide (30\%) and methanol $(100 \%)$. Endogenous peroxidase was blocked at room temperature and the samples were washed twice with PBS for 5 min each. The samples were incubated with bovine serum albuminblocking solution (added drop wise) at room temperature $\left(20^{\circ} \mathrm{C}\right)$ for $20 \mathrm{~min}$, and subsequently incubated with a primary antibody against $\alpha$-SMA $\left(1: 100\right.$; added drop wise) at $37^{\circ} \mathrm{C}$ for $1 \mathrm{~h}$. The samples were rinsed thrice with PBS for 2 min. The samples were then incubated with biotinlabeled secondary antibodies at $37^{\circ} \mathrm{C}$ for $20 \mathrm{~min}$, and subsequently rinsed thrice with PBS (2 min each). The samples were treated with horseradish peroxidase-labeled streptavidin working solution at $37^{\circ} \mathrm{C}$ for $20 \mathrm{~min}$, and subsequently washed with PBS (four times; $5 \mathrm{~min}$ each). The samples were then developed using a DAB kit, according to the manufacturer protocols. Briefly, the kit components $\mathrm{A}, \mathrm{B}$, and $\mathrm{C}$ were combined with distilled water, and the resulting solution was allowed to develop on a glass slide at room temperature. The slides were observed under an inverted microscope (Olympus Corporation) within 5-25 min. The slides were washed with distilled water to terminate the reaction and counter-stained with hematoxylin when necessary. Cells presenting brown- or tan-colored cytoplasmic regions were identified as being positively stained.

\section{Extraction and quantification of sample protein}

HSCs were lysed with a lysis buffer containing $8 \mathrm{M}$ urea, 4\% CHAPS (3-[(3-Cholamidopropyl)dimethylammonio]propanesulfonate), 1\% TATA-binding protein

Genetics and Molecular Research 15 (3): gmr.15038419 
(TBP), $0.2 \%$ BioLyte, and $0.001 \%$ bromophenol blue. The cells were incubated in an ice-bath at $4{ }^{\circ} \mathrm{C}$ with intermittent ultrasound and centrifuged at 22,000 $\mathrm{g}$ by a high-speed centrifuge (An Ting Scientific Instrument, Shanghai, China). The protein concentration in the cell supernatant was quantified by a standard Bradford assay.

\section{Detection of a-SMA expression in human HSCs by western blot}

A 10\% polyacrylamide gel was placed in an electrophoresis (Liu Yi Biotechnology, Beijing, China). The protein samples were loaded on the gel and separated by sodium dodecyl sulfate-polyacrylamide gel electrophoresis (SDS-PAGE) in Tris-glycine buffer. The gel was transferred to an electric transfer plate and the protein bands were transferred to a polyvinylidene difluoride (PVDF) membrane at $350 \mathrm{~mA}$ for $1 \mathrm{~h}$. The membrane was rinsed with Tris-buffered saline (TBS) for $10 \mathrm{~min}$, and incubated in blocking solution (TBS/T containing 5\% skim milk) for $1 \mathrm{~h}$ at room temperature. The membrane was then washed thrice in TBS/T ( 5 min each). The PVDF membrane was incubated with the $\alpha$-SMA primary antibody diluted in blocking solution $(1: 100)$ at $4^{\circ} \mathrm{C}$ overnight, rinsed thrice in TBS/T (5 min each), and subsequently incubated for $1 \mathrm{~h}$ with a horseradish peroxidase-conjugated secondary antibody diluted in blocking solution $(1: 3000)$ at $37^{\circ} \mathrm{C}$ in a shaker incubator. The membrane was rinsed thrice with TBS/T (5 min each) and then washed once with TBS. The PVDF membrane was visualized using an ECL kit. The film was exposed, developed, fixed, and analyzed using a BD transilluminator (BD Biosciences, Franklin Lakes, NJ, USA) and its accompanying software. GAPDH was used as the internal reference for semi-quantitative analysis.

\section{Detection of phospho-p38-MAPK protein levels by western blot}

A $10 \%$ polyacrylamide gel was prepared and placed in an electrophoresis (Liu Yi Biotechnology). The protein samples were loaded to the gel and separated by SDS-PAGE in Tris-glycine buffer. The gel was then transferred to an electric transfer plate and the protein bands were transferred to a PVDF membrane at $350 \mathrm{~mA}$ for $1 \mathrm{~h}$. The membrane was rinsed with TBS for $10 \mathrm{~min}$, and incubated in the blocking solution (TBS/T containing 5\% skim milk) for $1 \mathrm{~h}$ at room temperature. The membrane was then washed thrice in TBS/T (5-10 min each). The PVDF membrane was incubated with the phospho-p38-MAPK primary antibody diluted in blocking solution $(1: 1000)$ at $4^{\circ} \mathrm{C}$ overnight. Subsequently, it was rinsed thrice in TBS with Tween 20 (TBS/T) (5-10 min each), and incubated with a horseradish peroxidase-conjugated secondary antibody diluted in blocking solution $(1: 2000)$ at $37^{\circ} \mathrm{C}$ for $1 \mathrm{~h}$. The membrane was rinsed thrice with TBS/T (5-10 min each) and finally washed once with TBS. The PVDF membrane was visualized using an ECL kit. The film was exposed, developed, fixed, and analyzed using a BD transilluminator (BD Biosciences) and its accompanying software. GAPDH was used as the internal reference for semi-quantitative analysis.

\section{Statistical analysis}

Data were statistically analyzed using SPSS 17.0 (IBM, Armonk, NY, USA) and found to be normally distributed. Differences between two independent samples were determined using the Student $t$-test.

Genetics and Molecular Research 15 (3): gmr.15038419 


\section{RESULTS}

\section{Glucose-induced HSC activation is abrogated by p38-MAPK inhibition}

$\alpha$-SMA expression in human HSCs was analyzed in situ by the SABC method. The results are summarized in Figure 1. Cells stained a brown or tan were positive for $\alpha$-SMA expression. $\alpha$-SMA signals were stronger in the high-glucose group compared to the control group, while the signals in the blocking group were similar to those shown by the cells in the control group.

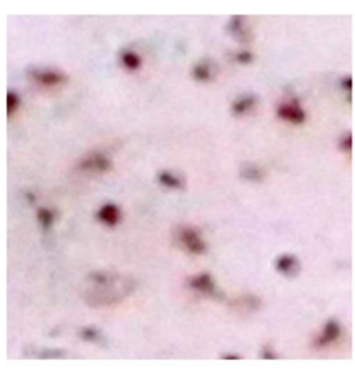

A

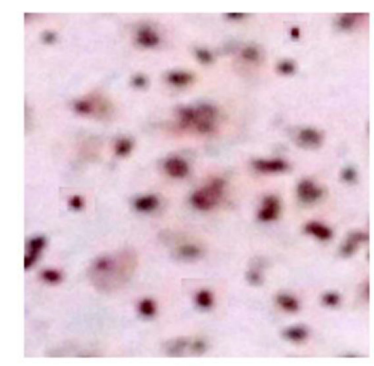

B

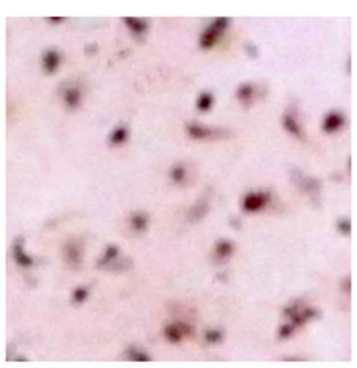

$\mathrm{C}$

Figure 1. Detection of $\alpha$-SMA expression in human hepatic stellate cells from each experimental group by the in situ SABC method (SABC, 200X). A. Control group; B. high-glucose group; and C. blocking group.

$\alpha$-SMA expression in human HSCs cultured normally, under high-glucose conditions, or under blocking conditions was analyzed by western blot, using GAPDH as the loading control (Figure 2). HSCs cultured under high-glucose conditions showed higher $\alpha$-SMA expression than HSCs in the control and blocking groups.

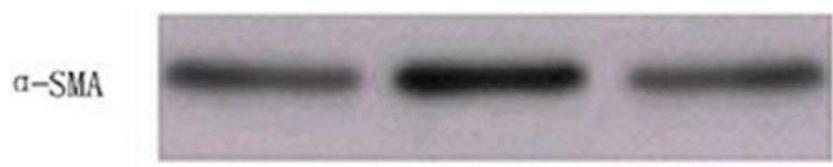

GAPDH

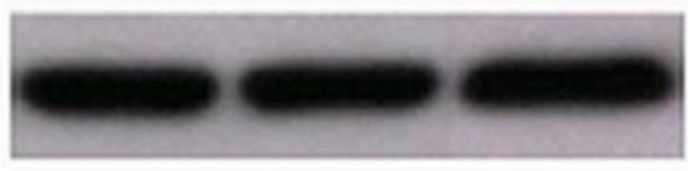

A

B

$\mathrm{C}$

Figure 2. Detection of $\alpha$-SMA protein expression in human hepatic stellate cells cultured under A. normal, B. highglucose, and C. blocking (SB203580-treated) conditions, by western blot analysis.

Phosphorylation of p38-MAPK induced by high levels of glucose by western blot was conducted to detect phospho-p38-MAPK expression in human HSCs cultured normally, under high-glucose conditions, and under blocking conditions, using GAPDH as the loading control (Figure 3). The phospho-p38-MAPK expression was higher in HSCs cultured under high-glucose conditions, than in HSCs belonging to the control and blocking groups.

Genetics and Molecular Research 15 (3): gmr.15038419 


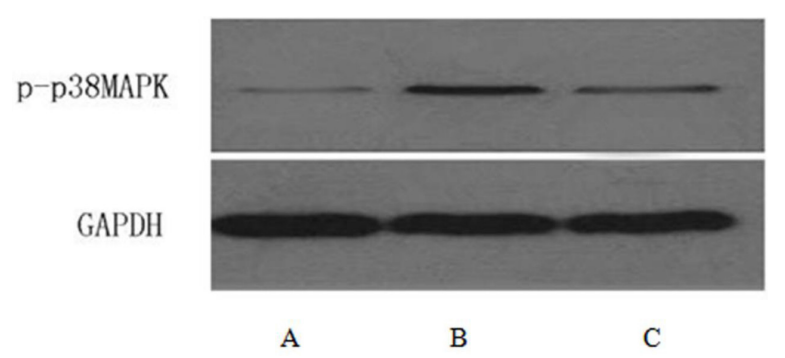

Figure 3. Detection of phospho-p38-MAPK protein expression in human hepatic stellate cells cultured under A. normal, B. high-glucose, and C. blocking (SB203580-treated) conditions by western blot analysis.

\section{Statistical analysis of experimental data}

Quantification of band intensities on the western blot indicated that phospho-p38MAPK expression was significantly higher in the high-glucose group than in the control group ( $\mathrm{P}<0.01$; Figure 3 and Table 1). Similar results were deduced from the $\alpha$-SMA band intensities $(\mathrm{P}<0.01$; Figure 2 and Table 1). Additionally, HSCs cultured under blocking conditions showed significantly lower phospho-p38-MAPK $(\mathrm{P}<0.01$; Figure 3 and Table 1$)$ and $\alpha$-SMA $(\mathrm{P}<0.01$; Figure 2 and Table 1$)$ expression levels than those cultured under highglucose conditions. These findings indicate that the p38-MAPK signaling pathway is closely related to HSC activation. High glucose-induced HSCs can be activated via intracellular p38MAPK signal transduction.

Table 1. Relative $\alpha$-smooth muscle actin ( $\alpha$-SMA) and phospho-p38-mitogen-activated protein kinase (p-p38MAPK) band intensities in each group (means \pm standard deviation (SD)).

\begin{tabular}{l|c|c}
\hline Group & $\alpha$-SMA/GAPDH protein (\%) & p-p38-MAPK/GAPDH protein (\%) \\
\hline Control group & $23.90 \pm 6.02$ & $8.13 \pm 4.955$ \\
\hline High-glucose group & $34.61 \pm 4.07^{* *}$ & $22.79 \pm 3.80^{* *}$ \\
\hline Blocking group & $26.48 \pm 4.41^{\Delta \Delta}$ & $10.66 \pm 4.15^{\Delta \Delta}$ \\
\hline
\end{tabular}

** $\mathrm{P}<0.01$ compared to the control group. ${ }^{\Delta \Delta} \mathrm{P}<0.01$ compared to the high-glucose group.

\section{DISCUSSION}

HSCs are activated by various external stimuli. Upon activation, they undergo morphological changes, ultimately transforming into myofibroblasts (MFB), which express various cytokines and cytokine receptors (Choi et al., 2010; Ikeda et al., 2012). These myofibroblasts synthesize extracellular matrix (ECM) components, including collagen I, collagen III, hyaluronic acid, and laminin (Iwaisako et al., 2012); therefore, they play a key role in initiating fibrosis. HSC activation results in a significant increase in the expression of type I collagen, with mRNA levels reaching 60 - to 70 -fold the basal levels. $\alpha$-SMA expression is a sign of HSC activation (Xu et al., 2009). Activated HSCs secrete several cytokines, including macrophage inflammatory protein-2 (MIP-2), monocyte chemoattractant protein-1 (MCP1 ), transforming growth factor (TGF)- $\beta$, hepatocyte growth factor (HGF), fibroblast growth factor (FGF), tumor necrosis factor (TNF)- $\alpha$, and interleukin (IL)-6, which are constituents of complex interaction networks that promote the development of hepatic fibrosis.

HSC activation is regulated by a complex signal-regulatory system, wherein multiple

Genetics and Molecular Research 15 (3): gmr.15038419 
cytokines and bioactive products released by liver cells, Kupffer cells, endothelial cells, and HSCs themselves are stimulated and released in vitro and in vivo. Additionally, many drugs are known to regulate HSC activation. Yang et al. (2008) and Sigala et al. (2013) reported that noradrenergic cells can induce HSC activation in a dose-dependent manner. Sugimoto et al. (2005) reported that high glucose conditions induce HSC activation via the MAPK signaling pathway. External signals can regulate HSC activation via the p38-MAPK signaling pathway, thereby regulating hepatic fibrosis. The p38-MAPK signaling pathway is involved in signal transduction cascades that are initiated by extracellular signals, which in turn play a significant role in several important intracellular biological processes. Phospho-p38-MAPK is formed by the dual phosphorylation of threonine and tyrosine sites; this is quickly transferred to the nucleus, and acts on its corresponding target to promote HSC activation (Kim and Choi, 2010). Activation of the $\mathrm{p} 38$-MAPK signaling pathway results in the regulation of HSC activity, reregulation of the balance between hepatic fibrosis-related factor matrix metalloproteinase-13 (MMP-13) and tissue inhibitor of metalloproteinase-1 (TIMP-1), and promotion of the development of liver fibrosis (Chen et al., 2010).

The aim of this study was to elucidate the relationship between the p38-MAPK signaling pathway and HSC activation. We discovered that blocking the p38-MAPK signaling pathway inhibits glucose-induced HSC activation (Wu et al., 2015). We observed a significant upregulation in phospho-p38-MAPK and $\alpha$-SMA expression in HSCs cultured under highglucose conditions; blocking the p38-MAPK signaling pathway was shown to inhibit this effect. This indicated a close relationship between the p38-MAPK signaling pathway and glucose-induced HSC activation. The p38-MAPK signal strength was also found to correspond to the level of HSC activation, while blocking the p38-MAPK signaling pathway reduced the high glucose-induced activation of HSCs. This study demonstrated that high levels of glucose promote the activation of HSCs via the p38-MAPK signaling pathway. These results lay the groundwork for future studies on HSC activation and liver fibrosis.

\section{Conflicts of interest}

The authors declare no conflict of interest.

\section{ACKNOWLEDGMENTS}

Research supported by the Zhejiang Provincial Health Science and Technology Program (\#2013KYA144)

\section{REFERENCES}

Brown MD and Sacks DB (2008). Compartmentalised MAPK pathways. Handb. Exp. Pharmacol. 186: 205-235. http:// dx.doi.org/10.1007/978-3-540-72843-6 9

Chen H, Ma C, Zhang YX and Ma H (2010). Adiponectin modulates MMP-13 and TIMP-1 expression via P38-MAPK pathway in rat hepatic stellate cell line. J. Cap. Med. Univ. 31: 304-309.

Choi SS, Syn WK, Karaca GF, Omenetti A, et al. (2010). Leptin promotes the myofibroblastic phenotype in hepatic stellate cells by activating the hedgehog pathway. J. Biol. Chem. 285: 36551-36560. http://dx.doi.org/10.1074/jbc. M110.168542

De Minicis S, Seki E, Uchinami H, Kluwe J, et al. (2007). Gene expression profiles during hepatic stellate cell activation in culture and in vivo. Gastroenterology 132: 1937-1946.http://dx.doi.org/10.1053/j.gastro.2007.02.033

Friedman SL (2008a). Hepatic fibrosis - overview. Toxicology 254: 120-129. http://dx.doi.org/10.1016/j.tox.2008.06.013

Genetics and Molecular Research 15 (3): gmr.15038419 
Friedman SL (2008b). Mechanisms of hepatic fibrogenesis. Gastroenterology 134: 1655-1669. http://dx.doi.org/10.1053/j. gastro.2008.03.003

Friedman SL (2008c). Hepatic stellate cells: protean, multifunctional, and enigmatic cells of the liver. Physiol. Rev. 88: 125-172. http://dx.doi.org/10.1152/physrev.00013.2007

Ikeda N, Murata S, Maruyama T, Tamura T, et al. (2012). Platelet-derived adenosine 5'-triphosphate suppresses activation of human hepatic stellate cell: In vitro study. Hepatol. Res. 42: 91-102.http://dx.doi.org/10.1111/j.1872034X.2011.00893.X

Iwaisako K, Brenner DA and Kisseleva T (2012). What's new in liver fibrosis? The origin of myofibroblasts in liver fibrosis. J. Gastroenterol. Hepatol. 27: 65-68. http://dx.doi.org/10.1111/j.1440-1746.2011.07002.x

Kim EK and Choi EJ (2010). Pathological roles of MAPK signaling pathways in human diseases. Biochim. Biophys. Acta. 1802: 396-405.http://dx.doi.org/10.1016/j.bbadis.2009.12.009

Kyriakis JM and Avruch J (2012). Mammalian MAPK signal transduction pathways activated by stress and inflammation: a 10-year update. Physiol. Rev. 92: 689-737.http://dx.doi.org/10.1152/physrev.00028.2011

Li JT, Liao ZX, Ping J, Xu D, et al. (2008). Molecular mechanism of hepatic stellate cell activation and antifibrotic therapeutic strategies. J. Gastroenterol. 43: 419-428. http://dx.doi.org/10.1007/s00535-008-2180-y

Sigala B, McKee C, Soeda J, Pazienza V, et al. (2013). Sympathetic nervous system catecholamines and neuropeptide Y neurotransmitters are upregulated in human NAFLD and modulate the fibrogenic function of hepatic stellate cells. PLoS One 8: e72928. http://dx.doi.org/10.1371/journal.pone.0072928

Sugimoto R, Enjoji M, Kohjima M, Tsuruta S, et al. (2005). High glucose stimulates hepatic stellate cells to proliferate and to produce collagen through free radical production and activation of mitogen-activated protein kinase. Liver Int. 25: 1018-1026. http://dx.doi.org/10.1111/j.1478-3231.2005.01130.x

Wu LK, Liu YC, Shi LL and Lu KD (2015). Glucagon-like peptide-1 receptor agonists inhibit hepatic stellate cell activation by blocking the p38-MAPK signaling pathway. Genet. Mol. Res. 29: 19087-19093. http://dx.doi.org/10.4238/2015. December. 29.17

Xu W, Wang LW, Shi JZ and Gong ZJ (2009). Effects of RNA interference targeting transforming growth factor-beta 1 on immune hepatic fibrosis induced by concanavalin A in mice. Hepatobiliary Pancreat. Dis. Int. 8: 300-308.

Yang Q, Zhang YW, Liu HY, Song YH, et al. (2008). Effects of norepinephrine on the proliferation and activation of rat hepatic stellate cells. Zhonghua Gan Zang Bing Za Zhi 16: 844-848.

Genetics and Molecular Research 15 (3): gmr.15038419 